\title{
SOME EXISTENCE AND UNIQUENESS RESULTS FOR FIRST-ORDER BOUNDARY VALUE PROBLEMS FOR IMPULSIVE FUNCTIONAL DIFFERENTIAL EQUATIONS WITH INFINITE DELAY IN FRÉCHET SPACES
}

JOHN R. GRAEF AND ABDELGHANI OUAHAB

Received 31 January 2006; Revised 19 May 2006; Accepted 28 May 2006

A recent nonlinear alternative for contraction maps in Fréchet spaces due to Frigon and Granas is used to investigate the existence and uniqueness of solutions to first-order boundary value problems for impulsive functional differential equations with infinite delay. An example to illustrate the results is included.

Copyright @ 2006 Hindawi Publishing Corporation. All rights reserved.

\section{Introduction}

This paper is devoted to the study of the existence and uniqueness of solutions of firstorder boundary value problems for impulsive functional and neutral impulsive functional differential equations with infinite delay. In particular, in Section 3, we will consider the class of first-order boundary value problem for functional differential equations with impulsive effects,

$$
\begin{gathered}
y^{\prime}(t)=f\left(t, y_{t}\right) \quad \text { a.e. } t \in J:=[0, \infty), t \neq t_{k}, k=1,2, \ldots, \\
y\left(t_{k}^{+}\right)-y\left(t_{k}^{-}\right)=I_{k}\left(y\left(t_{k}^{-}\right)\right), \quad t=t_{k}, k=1,2, \ldots, \\
A y_{0}-x_{\infty}=\phi(t), \quad t \in(-\infty, 0],
\end{gathered}
$$

where $f: J \times \mathscr{B} \rightarrow \mathbb{R}^{n}$ and $I_{k}: \mathbb{R}^{n} \rightarrow \mathbb{R}^{n}, k=1,2, \ldots$, are given functions, $\lim _{t \rightarrow \infty} y(t)=x_{\infty}$, $A \neq 1$, and $\phi \in \mathscr{B}$, which is called the phase space, will be defined later. Section 4 is devoted to the impulsive neutral functional differential equation with boundary conditions,

$$
\begin{gathered}
\frac{d}{d t}\left[y(t)-g\left(t, y_{t}\right)\right]=f\left(t, y_{t}\right), \quad t \in J, t \neq t_{k}, k=1,2, \ldots, \\
y\left(t_{k}^{+}\right)-y\left(t_{k}^{-}\right)=I_{k}\left(y\left(t_{k}^{-}\right)\right), \quad t=t_{k}, k=1,2, \ldots, \\
A y_{0}-x_{\infty}=\phi(t), \quad t \in(-\infty, 0]
\end{gathered}
$$

where $f, I_{k}, A, x_{\infty}$, and $\mathscr{B}$ are as in the problem (1.1)-(1.3) and $g: J \times \mathscr{B} \rightarrow \mathbb{R}^{n}$ is a given function. 
The notion of the phase space $\mathscr{B}$ plays an important role in the study of both the qualitative and quantitative theory of functional differential equations. A usual choice is a seminormed space satisfying suitable axioms as was introduced by Hale and Kato [18] (also see Kappel and Schappacher [21] and Schumacher [28]). For a detailed discussion on this topic, we refer the reader to Hino et al. [20]. For the case where the impulses are absent (i.e., $I_{k}=0, k=1,2, \ldots$ ), an extensive theory has been developed for the problem (1.1), (1.3), and we refer the reader to Corduneanu and Lakshmikantham [8], Hale and Kato [18], Hino et al. [20], Lakshmikantham et al. [23], and Shin [29].

Impulsive differential equations have become increasingly important in recent years as mathematical models of real-world processes and phenomena studied in control theory, physics, chemistry, population dynamics, biotechnology, and economics. There has been a significant development in impulse theory and this has been especially true in the area of impulsive differential equations with fixed moments; see, for example, the monographs of Baĭnov and Simeonov [3], Lakshmikantham et al. [22], and Samoılenko and Perestyuk [27], as well as the papers of Agur et al. [2], Ballinger and Liu [4], Benchohra et al. [5, 6], Franco et al. [9], and the references contained therein.

Boundary value problems on infinite intervals appear in many problems of practical interest, for example, in linear elasticity, nonlinear fluid flow, and foundation engineering (e.g., see, [1, 13, 16, 24-26]). Recently, fixed point arguments using such approaches as the Banach contraction principle, fixed point index theory, and monotone iterative technique have been applied to first- and second-order impulsive differential equations. We mention here the survey papers by Guo [12, 14, 15], Guo and Liu [17], Yan and Liu [30], and the references therein. Very recently, a nonlinear alternative due to Frigon and Granas [10] was applied to impulsive functional differential equation with variable times [11]; see the paper by Benchohra et al. [7] for first-order equations and Henderson and Ouahab [19] for second- and higher-order problems. Our goal here is to give existence and uniqueness results for the problems (1.1)-(1.3) and (1.4) above by using this nonlinear alternative for contraction maps.

\section{Preliminaries}

In this short section, we introduce notations and definitions that are used throughout the remainder of this paper. We let $C\left([0, b], \mathbb{R}^{n}\right)$ denote the Banach space of all continuous functions from $[0, b]$ into $\mathbb{R}^{n}$ with the norm

$$
\|y\|_{\infty}=\sup \{\|y(t)\|: 0 \leq t \leq b\}
$$

and we let $L^{1}\left([0, \infty), \mathbb{R}^{n}\right)$ be the Banach space of measurable functions $y:[0, \infty) \rightarrow \mathbb{R}^{n}$ that are Lebesgue integrable with the norm

$$
\|y\|_{L^{1}}=\int_{0}^{\infty}\|y(t)\| d t \quad \forall y \in L^{1}\left([0, \infty), \mathbb{R}^{n}\right) .
$$

For more details on the following notions, we refer the reader to Frigon and Granas [10]. Let $X$ be a Fréchet space with a family of seminorms $\left\{\|\cdot\|_{n}, n \in \mathbb{N}\right\}$. If $Y \subset X$, we say 
that $Y$ is bounded if for every $n \in \mathbb{N}$ there exists $M_{n}>0$ such that

$$
\|y\|_{n} \leq M_{n} \quad \forall y \in Y
$$

We associate to $X$ a sequence of Banach spaces $\left\{\left(X^{n},\|\cdot\|_{n}\right)\right\}$ as follows: for every $n \in \mathbb{N}$, we consider the equivalence relation $\sim_{n}$ defined by $x \sim_{n} y$ if and only if $\|x-y\|_{n}=0$ for all $x, y \in X$. We let $X^{n}=\left(X / \sim_{n},\|\cdot\|\right)$ denote the quotient space that is the completion of $X^{n}$ with respect to $\|\cdot\|_{n}$. To every $Y \subset X$, we associate a sequence $\left\{Y^{n}\right\}$ of subsets $Y^{n} \subset X^{n}$ as follows: for every $x \in X$, let $[x]_{n}$ denote the equivalence class of $x$ of subsets $X^{n}$, and we define $Y^{n}=\left\{[x]_{n}: x \in Y\right\}$. We let $\bar{Y}^{n}$, int ${ }_{n}\left(Y^{n}\right)$, and $\partial_{n} Y^{n}$, respectively, denote the closure, the interior, and the boundary of $Y^{n}$ with respect to $\|\cdot\|$ in $X^{n}$. We will assume that the family of seminorms $\left\{\|\cdot\|_{n}\right\}$ satisfies

$$
\|x\|_{1} \leq\|x\|_{2} \leq\|x\|_{3} \leq \cdots \quad \text { for every } x \in X .
$$

Next, we define what we mean by a contraction.

Definition 2.1. A function $f: X \rightarrow X$ is said to be a contraction if for each $n \in \mathbb{N}$ there exists $k_{n} \in(0,1)$ such that

$$
\|f(x)-f(y)\|_{n} \leq k_{n}\|x-y\|_{n} \quad \forall x, y \in X .
$$

The following nonlinear alternative will be used to prove our main results.

Theorem 2.2 (nonlinear alternative [10]). Let $X$ be a Fréchet space, let $Y \subset X$ be a closed subset in $X$, and let $N: Y \rightarrow X$ be a contraction such that $N(Y)$ is bounded. Then one of the following statements holds:

(C1) $N$ has a unique fixed point;

(C2) there exists $\lambda \in[0,1), n \in \mathbb{N}$, and $x \in \partial_{n} Y^{n}$ such that $\|x-\lambda N(x)\|_{n}=0$.

We will also need the following definition.

Definition 2.3. The map $f:[0, \infty) \times \mathscr{B} \rightarrow \mathbb{R}^{n}$ is said to be $L^{1}$-Carathéodory if

(i) $t \mapsto f(t, x)$ is measurable for each $x \in \mathscr{B}$;

(ii) $x \mapsto f(t, x)$ is continuous for almost all $t \in[0, \infty)$;

(iii) for each $q>0$ there exists $h_{q} \in L^{1}\left([0, \infty), \mathbb{R}_{+}\right)$such that

$$
\|f(t, x)\| \leq h_{q}(t) \quad \forall\|x\|_{\mathscr{B}} \leq q \text { and for almost all } t \in[0, \infty) .
$$

\section{Functional differential equations}

In order to define the phase space and a solution of the problem (1.1)-(1.3), we consider the space

$$
\begin{gathered}
\mathrm{PC}=\left\{y:(-\infty, \infty) \longrightarrow \mathbb{R}^{n} \mid y\left(t_{k}^{-}\right) \text {and } y\left(t_{k}^{+}\right) \text {exist with } y\left(t_{k}\right)=y\left(t_{k}^{-}\right),\right. \\
\left.A y(t)-x_{\infty}=\phi(t) \text { for } t \leq 0, y_{k} \in C\left(J_{k}, \mathbb{R}^{n}\right), k=1,2, \ldots\right\}
\end{gathered}
$$

where $y_{k}$ is the restriction of $y$ to $J_{k}=\left(t_{k}, t_{k+1}\right], k=1,2, \ldots$ In the remainder of this paper, we will assume that $\mathscr{B}$ satisfies the following properties. 
4 Impulsive functional differential equations

(A-1) If $y:(-\infty, \infty) \rightarrow \mathbb{R}^{n}$ and $y_{0} \in \mathscr{B}$, then for every $t$ in $[0, \infty)$, we have

(i) $y_{t}$ is in $\mathscr{B}$,

(ii) $\left\|y_{t}\right\|_{\mathscr{B}} \leq K(t) \sup \{|y(s)|: 0 \leq s \leq t\}+M(t)\left\|y_{0}\right\|_{\mathscr{B}}$, and

(iii) $|y(t)| \leq H\left\|y_{t}\right\|_{\mathscr{B}}$,

where $H \geq 0$ is a constant, $K:[0, \infty) \rightarrow[0, \infty)$ is continuous, $M:[0, \infty) \rightarrow[0, \infty)$ is locally bounded, and $H, K$, and $M$ are independent of $y$.

(A-2) For the function $y$ in (A-1), $y_{t}$ is a continuous function on $[0, \infty) \backslash\left\{t_{1}, t_{2}, \ldots\right\}$.

(A-3) The space $\mathscr{B}$ is complete.

Now set

$$
\begin{gathered}
B_{*}=\left\{y:(-\infty, \infty) \longrightarrow \mathbb{R}^{n}: y \in \mathrm{PC} \cap \mathscr{B}\right\}, \\
B_{k}=\left\{y \in B_{*}: \sup _{t \in J_{k}^{*}}|y(t)|<\infty\right\}, \quad \text { where } J_{k}^{*}=\left(-\infty, t_{k}\right] .
\end{gathered}
$$

Let $\|\cdot\|_{k}$ be the seminorm in $B_{k}$ defined by

$$
\|y\|_{k}=\left\|y_{0}\right\|_{\mathscr{B}}+\sup \left\{|y(s)|: 0 \leq s \leq t_{k}\right\}, \quad y \in B_{k}
$$

We will next define what we mean by a solution of (1.1)-(1.3).

Definition 3.1. A function $y$ is said to be a solution of the problem (1.1)-(1.3) if $y \in B_{*}$ and $y$ satisfies (1.1)-(1.3).

To prove our existence results for the problem (1.1)-(1.3), we first establish the following lemma.

Lemma 3.2. Let $f:[0, \infty) \rightarrow \mathbb{R}^{n}$ be a continuous function with $\int_{0}^{\infty} f(s) d s<\infty$. Then $y$ is a solution of the impulsive integral equation

$$
y(t)= \begin{cases}\frac{\phi(0)}{A(A-1)}+\frac{1}{A-1}\left[\int_{0}^{\infty} f\left(y_{s}\right) d s+\sum_{k=1}^{\infty} I_{k}\left(y\left(t_{k}^{-}\right)\right)\right]+\frac{\phi(t)}{A}, & t \in(-\infty, 0], \\ \frac{\phi(0)}{A-1}+\frac{1}{A-1}\left[\int_{0}^{\infty} f\left(y_{s}\right) d s+\sum_{k=1}^{\infty} I_{k}\left(y\left(t_{k}^{-}\right)\right)\right]+\int_{0}^{t} f\left(y_{s}\right) d s & \\ +\sum_{0<t_{k}<t} I_{k}\left(y\left(t_{k}^{-}\right)\right), & t \in[0, \infty),\end{cases}
$$

if and only if $y$ is a solution of the impulsive boundary value problem

$$
\begin{gathered}
y^{\prime}(t)=f\left(y_{t}\right), \quad t \in[0, \infty), t \neq t_{k}, k=1,2, \ldots, \\
y\left(t_{k}^{+}\right)-y\left(t_{k}^{-}\right)=I_{k}\left(y\left(t_{k}^{-}\right)\right), \quad k=1,2, \ldots, \\
A y_{0}-y_{\infty}=\phi(t), \quad t \in(-\infty, 0],
\end{gathered}
$$

where $\lim _{t \rightarrow \infty} y(t)=y_{\infty}$. 
Proof. Let $y$ be a solution of the impulsive integral equation (3.4). Then for $t \in[0, \infty)$ and $t \neq t_{k}, k=1,2, \ldots$, we have

$$
y(t)=\frac{\phi(0)}{A-1}+\frac{1}{A-1}\left[\int_{0}^{\infty} f\left(y_{s}\right) d s+\sum_{k=1}^{\infty} I_{k}\left(y\left(t_{k}^{-}\right)\right)\right]+\int_{0}^{t} f\left(y_{s}\right) d s+\sum_{0<t_{k}<t} I_{k}\left(y\left(t_{k}^{-}\right)\right) .
$$

Thus, $y^{\prime}(t)=f\left(y_{t}\right)$ for $t \in[0, \infty)$ and $t \neq t_{k}, k=1,2, \ldots$. From the definition of $y$, we see that

$$
y\left(t_{k}^{+}\right)-y\left(t_{k}^{-}\right)=I_{k}\left(y\left(t_{k}^{-}\right)\right) \quad \text { for } k=1,2, \ldots
$$

Finally, to see that $A y_{0}-y_{\infty}=\phi(t)$ for $t \in(-\infty, 0]$, note that

$$
\begin{gathered}
y_{\infty}=\frac{\phi(0)}{A-1}+\frac{A}{A-1}\left[\int_{0}^{\infty} f\left(y_{s}\right) d s+\sum_{k=1}^{\infty} I_{k}\left(y\left(t_{k}^{-}\right)\right)\right] \\
y_{0}=\frac{\phi(0)}{A(A-1)}+\frac{1}{A-1}\left[\int_{0}^{\infty} f\left(y_{s}\right) d s+\sum_{k=1}^{\infty} I_{k}\left(y\left(t_{k}^{-}\right)\right)\right]+\frac{\phi(t)}{A} .
\end{gathered}
$$

Hence,

$$
\begin{aligned}
A y_{0}-y_{\infty}= & \frac{\phi(0)}{A-1}+\frac{A}{A-1}\left[\int_{0}^{\infty} f\left(y_{s}\right) d s+\sum_{k=1}^{\infty} I_{k}\left(y\left(t_{k}^{-}\right)\right)\right]+\phi(t)-\frac{\phi(0)}{A-1} \\
& -\frac{A}{A-1}\left[\int_{0}^{\infty} f\left(y_{s}\right) d s+\sum_{k=1}^{\infty} I_{k}\left(y\left(t_{k}^{-}\right)\right)\right]=\phi(t) .
\end{aligned}
$$

Now let $y$ be a solution of the problem (3.5)-(3.7). Then,

$$
y^{\prime}(t)=f\left(y_{t}\right) \quad \text { for } t \in\left[0, t_{1}\right]
$$

and an integration from 0 to $t \in\left(0, t_{1}\right]$ yields

$$
y(t)-y(0)=\int_{0}^{t} y^{\prime}(s) d s=\int_{0}^{t} f\left(y_{s}\right) d s
$$

or

$$
y(t)=y(0)+\int_{0}^{t} f\left(y_{s}\right) d s
$$

for $t \in\left[0, t_{1}\right]$. If $t \in\left(t_{1}, t_{2}\right]$, then

$$
y\left(t_{1}^{-}\right)-y(0)+y(t)-y\left(t_{1}^{+}\right)=\int_{0}^{t_{1}} y^{\prime}(s) d s+\int_{t_{1}}^{t} y^{\prime}(s) d s=\int_{0}^{t} f\left(y_{s}\right) d s,
$$


6 Impulsive functional differential equations

so

$$
y(t)=y(0)+\int_{0}^{t} f\left(y_{s}\right) d s+I_{1}\left(y\left(t_{1}^{-}\right)\right) .
$$

Continuing this procedure, we obtain

$$
y(t)=y(0)+\int_{0}^{t} f\left(y_{s}\right) d s+\sum_{0<t_{k}<t} I_{k}\left(y\left(t_{k}^{-}\right)\right)
$$

for $t \in[0, \infty)$. Since $\lim _{t \rightarrow \infty} y(t)=y_{\infty}$, we have

$$
y_{\infty}=y(0)+\int_{0}^{\infty} f\left(y_{s}\right) d s+\sum_{k=1}^{\infty} I_{k}\left(y\left(t_{k}^{-}\right)\right) .
$$

From (3.7), we have $y_{\infty}=A y(0)-\phi(0)$, and so

$$
y(0)=\frac{\phi(0)}{A-1}+\frac{1}{A-1}\left[\int_{0}^{\infty} f\left(y_{s}\right) d s+\sum_{k=1}^{\infty} I_{k}\left(y\left(t_{k}^{-}\right)\right)\right] .
$$

Substituting (3.19) into (3.17), we obtain

$$
y(t)=\frac{\phi(0)}{A-1}+\frac{1}{A-1}\left[\int_{0}^{\infty} f\left(y_{s}\right) d s+\sum_{k=1}^{\infty} I_{k}\left(y\left(t_{k}^{-}\right)\right)\right]+\int_{0}^{t} f\left(y_{s}\right) d s+\sum_{0<t_{k}<t} I_{k}\left(y\left(t_{k}^{-}\right)\right)
$$

for $t \in[0, \infty)$.

From (3.7), (3.18), and (3.19), we have

$$
\begin{aligned}
y(t) & =\frac{\phi(t)}{A}+\frac{1}{A}\left[y(0)+\int_{0}^{\infty} f\left(y_{s}\right) d s+\sum_{k=1}^{\infty} I_{k}\left(y\left(t_{k}^{-}\right)\right)\right] \\
& =\frac{\phi(0)}{A(A-1)}+\frac{1}{A-1}\left[\int_{0}^{\infty} f\left(y_{s}\right) d s+\sum_{k=1}^{\infty} I_{k}\left(y\left(t_{k}^{-}\right)\right)\right]+\frac{\phi(t)}{A}
\end{aligned}
$$

for $t \in(-\infty, 0]$. This completes the proof of the lemma.

We are now ready to prove our existence and uniqueness result for the problem (1.1)(1.3)

Theorem 3.3. Let $f: J \times \mathscr{B} \rightarrow \mathbb{R}^{n}$ be an $L^{1}$-Carathéodory function and assume that

(H1) there exist constants $d_{k}>0, k=1,2, \ldots$, such that

$$
\left\|I_{k}(x)-I_{k}(\bar{x})\right\| \leq d_{k}\|x-\bar{x}\| \quad \text { for each } k=1,2, \ldots, \forall x, \bar{x} \in \mathbb{R}^{n} ;
$$

(H2) there exists a function $l \in L^{1}\left([0, \infty), \mathbb{R}^{n}\right)$ such that

$$
\|f(t, x)-f(t, \bar{x})\| \leq l(t)\|x-\bar{x}\|_{\mathscr{B}} \quad \text { for each } t \in[0, \infty), \forall x, \bar{x} \in \mathscr{B} ;
$$


(H3) there exist a function $p \in L^{1}\left([0, \infty), \mathbb{R}_{+}\right)$and positive constants $c_{k}, k=1,2, \ldots$, such that

$$
\begin{gathered}
\|f(t, u)\| \leq p(t) \quad \text { for }(t, u) \in[0, \infty) \times \mathscr{B}, \\
\left\|I_{k}(z)\right\| \leq c_{k} \quad \forall z \in \mathbb{R}^{n}, \\
\sum_{k=1}^{\infty} c_{k}<\infty .
\end{gathered}
$$

If $\sum_{k=1}^{\infty} d_{k}<1$, then the problem (1.1)-(1.3) has a unique solution.

Proof. We transform the problem (1.1)-(1.3) into a fixed point problem. Consider the operator $N: B_{*} \rightarrow B_{*}$ defined by

$$
N(y)(t)= \begin{cases}\frac{\phi(0)}{A(A-1)}+\frac{1}{A-1}\left[\int_{0}^{\infty} f\left(s, y_{s}\right) d s+\sum_{k=1}^{\infty} I_{k}\left(y\left(t_{k}^{-}\right)\right)\right]+\frac{\phi(t)}{A}, & t \in(-\infty, 0], \\ \frac{\phi(0)}{A-1}+\frac{1}{A-1}\left[\int_{0}^{\infty} f\left(s, y_{s}\right) d s+\sum_{k=1}^{\infty} I_{k}\left(y\left(t_{k}^{-}\right)\right)\right] & \\ +\int_{0}^{t} f\left(s, y_{s}\right) d s+\sum_{0<t_{k}<t} I_{k}\left(y\left(t_{k}^{-}\right)\right), & t \in[0, \infty) .\end{cases}
$$

It should be clear from Lemma 3.2 that the fixed points of $N$ are solutions of the problem (1.1)-(1.3). Let $x:(-\infty, \infty) \rightarrow \mathbb{R}^{n}$ be the function defined by

$$
x(t)= \begin{cases}\frac{\phi(0)}{A-1}+\frac{1}{A-1}\left[\int_{0}^{\infty} f\left(s, x_{s}\right) d s+\sum_{k=1}^{\infty} I_{k}\left(x\left(t_{k}^{-}\right)\right)\right] & \text {if } t \in[0, \infty), \\ \frac{\phi(0)}{A(A-1)}+\frac{1}{A-1}\left[\int_{0}^{\infty} f\left(s, x_{s}\right) d s+\sum_{k=1}^{\infty} I_{k}\left(x\left(t_{k}^{-}\right)\right)\right]+\frac{\phi(t)}{A} & \text { if } t \in(-\infty, 0] .\end{cases}
$$

Then,

$$
x_{0}=\frac{\phi(0)}{A-1}+\frac{1}{A-1}\left[\int_{0}^{\infty} f\left(s, x_{s}\right) d s+\sum_{k=1}^{\infty} I_{k}\left(x\left(t_{k}^{-}\right)\right)\right] .
$$

For each $z \in C\left([0, \infty), \mathbb{R}^{n}\right)$ with $z(0)=0$, denote by $\bar{z}$ the function given by

$$
\bar{z}(t)= \begin{cases}z(t) & \text { if } t \in[0, \infty) \\ 0 & \text { if } t \in(-\infty, 0]\end{cases}
$$

If $y$ satisfies the integral equation

$$
y(t)=\frac{\phi(0)}{A-1}+\frac{1}{A-1}\left[\int_{0}^{\infty} f\left(s, y_{s}\right) d s+\sum_{k=1}^{\infty} I_{k}\left(y\left(t_{k}^{-}\right)\right)\right]+\int_{0}^{t} f\left(s, y_{s}\right) d s+\sum_{0<t_{k}<t} I_{k}\left(y\left(t_{k}^{-}\right)\right),
$$


we can decompose $y$ into $y(t)=\bar{z}(t)+x(t), 0 \leq t<\infty$, which implies that $y_{t}=\bar{z}_{t}+x_{t}$ for $0 \leq t<\infty$, and $z$ satisfies

$$
z(t)=\int_{0}^{t} f\left(s, \bar{z}_{s}+x_{s}\right) d s+\sum_{0<t_{k}<t} I_{k}\left(\bar{z}\left(t_{k}^{-}\right)+x\left(t_{k}^{-}\right)\right) .
$$

Let

$$
B_{*}^{k}=\left\{z \in B_{k}: z_{0}=0\right\}
$$

For any $z \in B_{*}^{k}$, we have

$$
\|z\|_{k}=\left\|z_{0}\right\|_{\Re}+\sup \left\{\|z(s)\|: 0 \leq s \leq t_{k}\right\}=\sup \left\{\|z(s)\|: 0 \leq s \leq t_{k}\right\} .
$$

Thus $\left(B_{*}^{k},\|\cdot\|_{k}\right)$ is a Banach space. If we set

$$
C_{0}=\left\{z \in B_{*}: z_{0}=0\right\}
$$

with the Bielecki-type norm on $B_{*}^{k}$ defined by

$$
\|z\|_{B_{*}^{k}}=\max \left\{\|z(t)\| e^{-\tau \hat{l}(t)}: t \in\left[0, t_{k}\right]\right\},
$$

where $\hat{l}(t)=\int_{0}^{t} \tilde{l}(s) d s, \tilde{l}(t)=K_{k} l(t), K_{k}=\sup \left\{|K(t)|: t \in\left[0, t_{k}\right]\right\}, k=1,2, \ldots$, and $\tau>0$ is a constant, then $C_{0}$ is a Fréchet space with the family of seminorms $\|\cdot\|_{B_{*}^{k}}$.

Let the operator $P: C_{0} \rightarrow C_{0}$ be defined by

$$
(P z)(t)= \begin{cases}0, & t \leq 0, \\ \int_{0}^{t} f\left(s, \bar{z}_{s}+x_{s}\right) d s+\sum_{0<t_{k}<t} I_{k}\left(\bar{z}\left(t_{k}^{-}\right)+x\left(t_{k}^{-}\right)\right), & t \in[0, \infty) .\end{cases}
$$

Clearly, the operator $N$ having a fixed point is equivalent to the operator $P$ having one, and so we turn our attention to proving that $P$ does have a fixed point. We will use the nonlinear alternative given in Theorem 2.2 to show this.

Let $z$ be a possible solution of the problem $z=\lambda P(z)$ for some $0<\lambda<1$. Then, for each $t \in[0, \infty)$,

$$
z(t)=\lambda\left[\int_{0}^{t} f\left(s, \bar{z}_{s}+x_{s}\right) d s+\sum_{0<t_{k}<t} I_{k}\left(\bar{z}\left(t_{k}^{-}\right)+x\left(t_{k}^{-}\right)\right)\right],
$$

so by (H3), we have

$$
\|z(t)\|<\int_{0}^{t} p(s) d s+\sum_{i=1}^{i=k} I_{i}\left(\bar{z}\left(t_{i}\right)+x\left(t_{i}\right)\right) \mid \leq \int_{0}^{t_{k}} p(s) d s+\sum_{i=1}^{i=k} c_{i}:=M_{k}^{*} .
$$

Hence,

$$
\sup \left\{\|z(t)\|: t \in\left[0, t_{k}\right]\right\}<M_{k}^{*}
$$


Let

$$
Y=\left\{z \in C_{0} \mid \sup \left\{\|z\|_{B_{*}^{k}}: 0 \leq t \leq t_{k}\right\} \leq M_{k}^{*} \forall k=1,2, \ldots\right\}
$$

Clearly, $Y$ is a closed subset of $C_{0}$. We will show that $P: Y \rightarrow B_{*}^{k}$ is a contraction map. To see this, consider $z, z^{*} \in Y$; then for each $t \in\left[0, t_{k}\right]$ and $k=1,2, \ldots$, we have

$$
\begin{aligned}
& \left\|P(z)(t)-P\left(z^{*}\right)(t)\right\| \leq \int_{0}^{t}\left\|f\left(s, \bar{z}_{s}+x_{s}\right)-f\left(s, \bar{z}_{s}^{*}+x_{s}\right)\right\| d s \\
& +\sum_{i=1}^{i=k}\left\|I_{i}\left(\bar{z}\left(t_{i}^{-}\right)+x\left(t_{i}^{-}\right)\right)-I_{i}\left(\bar{z}^{*}\left(t_{i}^{-}\right)+x\left(t_{i}^{-}\right)\right)\right\| \\
& \leq \int_{0}^{t} l(s)\left\|\bar{z}_{s}-\bar{z}_{s}^{*}\right\|_{\Re} d s+\sum_{i=1}^{i=k} d_{i}\left\|z\left(t_{i}^{-}\right)-z^{*}\left(t_{i}^{-}\right)\right\| \\
& \leq \int_{0}^{t} l(s) K_{k} \sup _{s \in[0, t]}\left\|z(s)-z^{*}(s)\right\| d s+\sum_{i=1}^{i=k} d_{i}\left\|z\left(t_{i}^{-}\right)-z^{*}\left(t_{i}^{-}\right)\right\| \\
& \leq \int_{0}^{t} \tilde{l}(s) e^{\tau \hat{l}(s)} e^{-\tau \hat{l}(s)} \sup _{s \in[0, t]}\left\|z(s)-z^{*}(s)\right\| d s \\
& +\sum_{i=1}^{i=k} d_{i} e^{\tau \hat{\imath}(t)} e^{-\tau \hat{\imath}(t)} \sup _{s \in\left[0, t_{k}\right]}\left\|z(s)-z^{*}(s)\right\| \\
& =\int_{0}^{t} \tilde{l}(s) e^{\tau \hat{l}(s)} d s\left\|z-z^{*}\right\|_{B_{*}^{k}}+\sum_{i=1}^{i=k} d_{i} e^{\tau \hat{l}(t)}\left\|z-z^{*}\right\|_{B_{*}^{k}} \\
& =\frac{1}{\tau} \int_{0}^{t}\left(e^{\tau \hat{l}(s)}\right)^{\prime} d s\left\|z-z^{*}\right\|_{B_{*}^{k}}+\sum_{i=1}^{i=k} d_{i} e^{\tau \hat{l}(t)}\left\|z-z^{*}\right\|_{B_{*}^{k}} \\
& \leq \frac{1}{\tau} e^{\tau \hat{l}(t)} d s\left\|z-z^{*}\right\|_{B_{*}^{k}}+\sum_{i=1}^{i=k} d_{i} e^{\tau \hat{l}(t)}\left\|z-z^{*}\right\|_{B_{*}^{k}}
\end{aligned}
$$

Thus,

$$
e^{-\hat{\imath}(t)}\left\|P(z)(t)-P\left(z^{*}\right)(t)\right\| \leq \frac{1}{\tau}\left\|z-z^{*}\right\|_{B_{*}^{k}}+\sum_{i=1}^{i=k} d_{i}\left\|z-z^{*}\right\|_{B_{*}^{k}}=\left(\frac{1}{\tau}+\sum_{i=1}^{i=k} d_{i}\right)\left\|z-z^{*}\right\|_{B_{*}^{k}} .
$$

Therefore,

$$
\left\|P(z)-P\left(z^{*}\right)\right\|_{B_{*}^{k}} \leq\left(\frac{1}{\tau}+\sum_{i=1}^{i=k} d_{i}\right)\left\|z-z_{*}\right\|_{B_{*}^{k}}
$$

which shows that if $\tau$ is large enough, then $P$ is a contraction. From the choice of $Y$, there is no $z \in \partial Y^{n}$ such that $z=\lambda P(z)$ for some $\lambda \in(0,1)$. As a consequence of Theorem 2.2, 
we conclude that $P$ has a unique fixed point. In turn, this implies that the operator $N$ has a unique fixed point that is a solution to (1.1)-(1.3). This completes the proof of the theorem.

\section{Neutral functional differential equations}

This section is concerned with the existence of solutions to the boundary value problem for first-order neutral functional differential equations with infinite delay and impulses given in (1.4). Our main result is as follows.

Theorem 4.1. Let $f: J \times \mathscr{B} \rightarrow \mathbb{R}^{n}$ be an $L^{1}$-Carathéodory function. In addition to (H1)(H3), assume that $M(0)<1$ and

(B) there exist constants $c_{1}^{*}, c_{2}^{*} \geq 0$, and $\bar{d}_{k}>0, k=1,2, \ldots$, such that

$$
\begin{gathered}
\|g(t, u)\| \leq c_{1}^{*}\|u\|_{\mathscr{B}}+c_{2}^{*}, \quad t \in[0, \infty), u \in \mathscr{B}, c_{1}^{*} K_{k}<1, \\
\|g(t, u)-g(t, \bar{u})\| \leq \bar{d}_{k}\|u-\bar{u}\|_{\mathscr{B}} \quad \text { for } t \in\left[0, t_{k}\right],
\end{gathered}
$$

where

$$
K_{k}=\sup \left\{|K(t)|: t \in\left[0, t_{k}\right]\right\}, \quad k=1,2, \ldots
$$

If $\sum_{k=1}^{\infty}\left[d_{k}+\bar{d}_{k} K_{k}\right]<1$, then the problem (1.4) has a unique solution.

Proof. We proceed similarly to what we did in the proof of Theorem 3.3 by defining the operators $N_{1}: B_{*} \rightarrow B_{*}$ and $P_{1}: C_{0} \rightarrow C_{0}$ by

$$
\begin{aligned}
& N_{1}(y)(t)= \begin{cases}g(0, \phi(0))-g(t, \phi(t))+\frac{\phi(0)}{A(A-1)}+\frac{\phi(t)}{A} & \\
+\frac{1}{A-1}\left[\int_{0}^{\infty} f\left(s, y_{s}\right) d s+\sum_{k=1}^{\infty} I_{k}\left(y\left(t_{k}^{-}\right)\right)\right], & t \in(-\infty, 0], \\
g(0, \phi)-g\left(t, y_{s}\right)+\frac{1}{A-1}\left[\int_{0}^{\infty} f\left(s, y_{s}\right) d s+\sum_{k=1}^{\infty} I_{k}\left(y\left(t_{k}^{-}\right)\right)\right] & t \in[0, \infty), \\
+\frac{\phi(0)}{A-1}+\int_{0}^{t} f\left(s, y_{s}\right) d s+\sum_{0<t_{k}<t} I_{k}\left(y\left(t_{k}^{-}\right)\right), & t\end{cases} \\
& \left(P_{1} z\right)(t)= \begin{cases}0, & t \leq 0, \\
g(0, \phi)-g\left(t, \bar{z}_{t}+x_{t}\right)+\int_{0}^{t} f\left(s, \bar{z}_{s}+x_{s}\right) d s+\sum_{0<t_{k}<t} I_{k}\left(\bar{z}\left(t_{k}^{-}\right)+x\left(t_{k}^{-}\right)\right), & t \in[0, \infty),\end{cases}
\end{aligned}
$$

where $\bar{z}_{t}$ and $x_{t}$ are defined similar to the way they are in the proof of Theorem 3.3. In order to apply the nonlinear alternative, Theorem 2.2, we first obtain a priori estimates for the solutions of the integral equation

$$
z(t)=\lambda\left[g(0, \phi)-g\left(t, \bar{z}_{t}+x_{t}\right)+\int_{0}^{t} f\left(s, \bar{z}_{s}+x_{s}\right) d s+\sum_{0<t_{k}<t} I_{k}\left(\bar{z}\left(t_{k}^{-}\right)+x\left(t_{k}^{-}\right)\right)\right]
$$


for any $\lambda \in(0,1)$. We have

$$
\begin{aligned}
\|z(t)\|< & \|g(0, \phi(0))\|+\left\|g\left(t, \bar{z}_{t}+x_{t}\right)\right\|+\int_{0}^{t} p(s) d s+\sum_{0<t_{k}<t}\left\|I_{k}\left(\bar{z}\left(t_{k}^{-}\right)+x\left(t_{k}^{-}\right)\right)\right\| \\
\leq & \|g(0, \phi)\|+c_{1}^{*}\left\|\bar{z}_{t}+x_{t}\right\|_{\mathscr{B}}+c_{2}^{*}+\int_{0}^{t} p(s) d s+\sum_{i=1}^{i=k} c_{i} \\
\leq & \|g(0, \phi(0))\|+c_{1}^{*} K(t) \sup _{s \in[0, t]}|z(s)|+c_{1}^{*} K(t) \sup _{s \in[0, t]}|x(s)|+c_{1}^{*} M(t)\left\|x_{0}\right\|_{\Re} \\
& +c_{2}^{*}+\int_{0}^{t} p(s) d s+\sum_{i=1}^{k} c_{i} \leq\|g(0, \phi(0))\|+c_{1}^{*} K_{k} \sup _{s \in[0, t]}|z(s)| \\
& +K_{k} c_{1}^{*}\|x\|_{\infty}+M_{k} c_{1}^{*}\left\|x_{0}\right\|_{\mathscr{B}}+c_{2}^{*}+\int_{0}^{t} p(s) d s+\sum_{i=1}^{k} c_{i},
\end{aligned}
$$

where

$$
M_{k}=\sup \left\{M(t): t \in\left[0, t_{k}\right]\right\} .
$$

Hence,

$$
\|z\|_{\infty}<\|g(0, \phi)\|+c_{1}^{*} K_{k}\|z\|_{\infty}+K_{k} c_{1}^{*}\|x\|_{\infty}+M_{k} c_{1}^{*}\left\|x_{0}\right\|_{\mathscr{B}}+c_{2}^{*}+\int_{0}^{\infty} p(s) d s+\sum_{i=1}^{k} c_{i} .
$$

Now,

$$
\left\|x_{0}\right\|_{\Re} \leq K(0)|x(0)|+M(0)|| x_{0} \|_{\Re},
$$

so

$$
\left\|x_{0}\right\|_{\mathscr{B}} \leq \frac{K(0)}{1-M(0)}|x(0)|
$$

and since

$$
\|x\|_{\infty}:=\sup _{t \in\left[0, t_{k}\right]}|x(t)| \leq \frac{|\phi(0)|}{A-1}+\frac{1}{A-1}\left[\|p\|_{L^{1}}+\sum_{k=1}^{\infty} c_{k}\right]:=\ell
$$

we then have

$$
\|z\|_{\infty}<\frac{1}{1-c_{1}^{*} K_{k}}\left[\|g(0, \phi)\|+c_{2}^{*}+K_{k} c_{1}^{*} \ell+M_{k} c_{1}^{*} \frac{K(0)}{1-M(0)} \ell+\|p\|_{L^{1}}+\sum_{i=1}^{i=k} c_{i}\right]:=\bar{M}_{k} .
$$


12 Impulsive functional differential equations

Now set

$$
Y_{1}=\left\{z \in C_{0}: \sup \left\{\|z(t)\|: 0 \leq t \leq t_{k}\right\} \leq \bar{M}_{k} \forall k=1,2, \ldots\right\}
$$

Clearly, $Y_{1}$ is a closed subset of $C_{0}$. To show that $P_{1}$ is a contraction, let $z, z^{*} \in Y_{1}$. Then, for each $t \in\left[0, t_{k}\right]$ and $k=1,2, \ldots$, we have

$$
\begin{aligned}
& \left\|P_{1}(z)(t)-P_{1}\left(z^{*}\right)(t)\right\| \\
& \leq\left\|g\left(t, \bar{z}_{t}+x_{t}\right)-g\left(t, \bar{z}_{t}^{*}+x_{t}\right)\right\|+\int_{0}^{t}\left\|f\left(s, \bar{z}_{s}+x_{s}\right)-f\left(s, \bar{z}_{s}^{*}+x_{s}\right)\right\| d s \\
& +\sum_{i=1}^{i=k}\left\|I_{i}\left(\bar{z}\left(t_{i}^{-}\right)+x\left(t_{i}^{-}\right)\right)-I_{i}\left(\bar{z}^{*}\left(t_{i}^{-}\right)+x\left(t_{i}^{-}\right)\right)\right\| \leq \bar{d}_{k}\left\|\bar{z}_{t}-z_{t}^{*}\right\|_{\mathscr{B}} \\
& +\int_{0}^{t} l(s)\left\|\bar{z}_{s}-\bar{z}_{s}^{*}\right\|_{\Re} d s+\sum_{i=1}^{i=k} d_{i}\left\|z\left(t_{i}^{-}\right)-z^{*}\left(t_{i}^{-}\right)\right\| \leq \bar{d}_{k} K_{k} \sup _{t \in\left[0, t_{k}\right]}\left\|z(t)-z^{*}(t)\right\| \\
& +\int_{0}^{t} l(s) K_{k} \sup _{\tau \in[0, s]}\left\|z(\tau)-z^{*}(\tau)\right\| d s+\sum_{i=1}^{i=k} d_{i}\left\|z\left(t_{i}^{-}\right)-z^{*}\left(t_{i}^{-}\right)\right\| \\
& \leq \bar{d}_{k} K_{k} e^{\tau \hat{l}(t)} e^{-\tau \hat{\imath}(t)} \sup _{t \in\left[0, t_{k}\right]}\left\|z(t)-z^{*}(t)\right\|+\int_{0}^{t} \tilde{l}(s) e^{\tau \hat{\imath}(s)} e^{-\tau \hat{l}(s)} \sup _{\tau \in[0, s]}\left\|z(\tau)-z^{*}(\tau)\right\| d s \\
& +\sum_{i=1}^{i=k} d_{i} e^{\tau \hat{\imath}(t)} e^{-\tau \hat{l}(t)} \sup _{s \in\left[0, t_{k}\right]}\left\|z(s)-z^{*}(s)\right\| \leq \bar{d}_{k} K_{k} e^{\tau \hat{l}(t)}\left\|z-z^{*}\right\|_{B_{*}^{k}} \\
& +\int_{0}^{t} \tilde{l}(s) e^{\tau \hat{l}(s)} d s\left\|z-z^{*}\right\|_{B_{*}^{k}}+\sum_{i=1}^{i=k} d_{i} e^{\tau \hat{l}(t)}\left\|z-z^{*}\right\|_{B_{*}^{k}}=\bar{d}_{k} K_{k} e^{\tau \hat{\imath}(t)}\left\|z-z^{*}\right\|_{B_{*}^{k}} \\
& +\frac{1}{\tau} \int_{0}^{t}\left(e^{\tau \hat{\imath}(s)}\right)^{\prime} d s\left\|z-z^{*}\right\|_{B_{*}^{k}}+\sum_{i=1}^{i=k} d_{i} e^{\tau \hat{l}(t)}\left\|z-z^{*}\right\|_{B_{*}^{k}} \leq \bar{d}_{k} K_{k} e^{\tau \hat{l}(t)}\left\|z-z^{*}\right\|_{B_{*}^{k}} \\
& +\frac{1}{\tau} e^{\tau \hat{l}(t)} d s\left\|z-z^{*}\right\|_{B_{*}^{k}}+\sum_{i=1}^{i=k} d_{i} e^{\tau \hat{l}(t)}\left\|z-z^{*}\right\|_{B_{*}^{k}} .
\end{aligned}
$$

Thus,

$$
e^{-\tau \hat{\imath}(t)}\left\|P_{1}(z)(t)-P_{1}\left(z^{*}\right)(t)\right\| \leq\left(\frac{1}{\tau}+\sum_{i=1}^{i=k} d_{i}+\bar{d}_{k} K_{k}\right)\left\|z-z^{*}\right\|_{B_{*}^{k}} .
$$

Therefore,

$$
\left\|P_{1}(z)-P_{1}\left(z^{*}\right)\right\|_{B_{*}^{k}} \leq\left(\frac{1}{\tau}+\sum_{i=1}^{i=k} d_{i}+\bar{d}_{k} K_{k}\right)\left\|z-z_{*}\right\|_{B_{*}^{k}},
$$


and again if $\tau$ is large enough, $P_{1}$ is a contraction. From the choice of $Y_{1}$, there is no $z \in \partial Y_{1}^{n}$ such that $z=\lambda P_{1}(z)$ for some $\lambda \in(0,1)$. As a consequence of the nonlinear alternative (Theorem 2.2), we see that $P_{1}$ has a unique fixed point which again leads to the existence of a unique solution to (1.4).

\section{Example}

In this section we give an example to illustrate the usefulness of our main results. Consider the problem

$$
\begin{gathered}
y^{\prime}(t)=\frac{e^{-\gamma t}\left|y_{t}\right|}{(t+1)(t+2)\left(1+\left|y_{t}\right|\right)}, \quad \text { a.e. } t \in J:=[0, \infty)-\left\{t_{1}, t_{2}, \ldots\right\}, \\
y\left(t_{k}^{+}\right)-y\left(t_{k}^{-}\right)=b_{k} y\left(t_{k}^{-}\right), \quad k=1,2, \ldots, \\
y(t)=\phi(t), \quad t \in(-\infty, 0],
\end{gathered}
$$

where $\gamma>0$ is a constant and $b_{k}>0$ for $k=1,2, \ldots$. Let

$\mathscr{D}=\left\{\psi:(-\infty, 0] \longrightarrow \mathbb{R}^{n} \mid \psi\right.$ is continuous everywhere except for a countable number of points $\bar{t}$ at which $\left.\psi\left(\bar{t}^{-}\right), \psi\left(\bar{t}^{+}\right) \exists, \psi\left(\bar{t}^{-}\right)=\psi(\bar{t})\right\}$.

Let

$$
B_{\gamma}=\left\{y \in \mathscr{D}: \lim _{\theta \rightarrow-\infty} e^{\gamma \theta} y(\theta) \text { exists in } \mathbb{R}^{n}\right\}
$$

with the norm in $B_{\gamma}$ given by

$$
\|y\|_{\gamma}=\sup _{-\infty<\theta \leq 0} e^{\gamma \theta}\|y(\theta)\|
$$

Let $y:(-\infty, \infty) \rightarrow \mathbb{R}^{n}$ be such that $y_{0} \in B_{\gamma}$; then

$$
\lim _{\theta \rightarrow-\infty} e^{\gamma \theta} y_{t}(\theta)=\lim _{\theta \rightarrow-\infty} e^{\gamma \theta} y(t+\theta)=\lim _{\theta \rightarrow-\infty} e^{\gamma(\theta-t)} y(\theta)=e^{-\gamma t} \lim _{\theta \rightarrow-\infty} e^{\gamma \theta} y_{0}(\theta)<\infty .
$$

Hence, $y_{t} \in B_{\gamma}$.

Next, we prove that $|y(t)| \leq H\left\|y_{t}\right\|_{B_{\gamma}}$ and

$$
\left\|y_{t}\right\| \leq K(t) \sup \{\|y(s)\|: 0 \leq s \leq t\}+M(t)\left\|y_{0}\right\|_{\gamma},
$$

where $\left\|y_{t}(\theta)\right\|=\|y(t+\theta)\|, K(t) \equiv M(t) \equiv 1$, and $H=1$. Now, if $\theta+t \leq 0$, we have

$$
\left\|y_{t}(\theta)\right\| \leq \sup \{\|y(s)\|:-\infty<s \leq 0\} .
$$

For $t+\theta \geq 0$, we see that

$$
\left\|y_{t}(\theta)\right\| \leq \sup \{\|y(s)\|: 0<s \leq t\} .
$$


Thus, for all $t+\theta \in \mathbb{R}$, we obtain

$$
\left\|y_{t}(\theta)\right\| \leq \sup \{\|y(s)\|:-\infty<s \leq 0\}+\sup \{\|y(s)\|: 0 \leq s \leq t\} .
$$

Then,

$$
\left\|y_{t}\right\|_{\gamma} \leq\left\|y_{0}\right\|_{\gamma}+\sup \{\|y(s)\|: 0 \leq s \leq t\}
$$

Now $\left(B_{\gamma},\|\cdot\|\right)$ is a Banach space and $B_{\gamma}$ will serve as the phase space for our problem. We see that the function $f$ satisfies

$$
\begin{gathered}
|f(t, x)|=\frac{e^{-\gamma t}|x|}{(t+1)(t+2)(1+|x|)} \leq \frac{1}{(t+1)(t+2)}=p(t), \\
\int_{0}^{\infty} p(t) d t=\int_{0}^{\infty} \frac{1}{(t+1)(t+2)} d t=\ln 2 .
\end{gathered}
$$

Let $x, y \in B_{y}$; then,

$$
\begin{aligned}
|f(t, x)-f(t, y)| & =\frac{e^{-\gamma t}}{(t+1)(t+2)}\left|\frac{|x|}{1+|x|}-\frac{|y|}{1+|y|}\right|=\frac{e^{-\gamma t}|| x|-| y||}{(t+1)(t+2)(1+|x|)(1+|y|)} \\
& \leq \frac{e^{-\gamma t}|x-y|}{(t+1)(t+2)(1+|x|)(1+|y|)} \leq \frac{\|x-y\|_{B_{\gamma}}}{(t+1)(t+2)} .
\end{aligned}
$$

Hence, the hypotheses of Theorem 3.3 are satisfied, so if $\sum_{k=1}^{\infty} b_{k}<1$, then the problem (5.1) has a unique solution.

\section{Acknowledgment}

The research of J. R. Graef was supported in part by the the Office of Academic and Research Computing Services of the University of Tennessee at Chattanooga.

\section{References}

[1] R. P. Agarwal and D. O'Regan, Infinite Interval Problems for Differential, Difference and Integral Equations, Kluwer Academic, Dordrecht, 2001.

[2] Z. Agur, L. Cojocaru, G. Mazaur, R. M. Anderson, and Y. L. Danon, Pulse mass measles vaccination across age cohorts, Proceedings of the National Academy of Sciences of the United States of America 90 (1993), 11698-11702.

[3] D. D. Bănov and P. S. Simeonov, Systems with Impulse Effect. Stability, Theory and Applications, Ellis Horwood Series: Mathematics and Its Applications, Ellis Horwood, Chichester, 1989.

[4] G. Ballinger and X. Liu, Existence and uniqueness results for impulsive delay differential equations, Dynamics of Continuous, Discrete and Impulsive Systems 5 (1999), no. 1-4, 579-591.

[5] M. Benchohra, J. Henderson, and S. K. Ntouyas, An existence result for first-order impulsive functional differential equations in Banach spaces, Computers \& Mathematics with Applications 42 (2001), no. 10-11, 1303-1310.

[6] _ Impulsive neutral functional differential equations in Banach spaces, Applicable Analysis 80 (2001), no. 3-4, 353-365. 
[7] M. Benchohra, J. Henderson, S. K. Ntouyas, and A. Ouahab, Impulsive functional differential equations with variable times and infinite delay, International Journal of Applied Mathematical Sciences 2 (2005), no. 1, 130-148.

[8] C. Corduneanu and V. Lakshmikantham, Equations with unbounded delay: a survey, Nonlinear Analysis 4 (1980), no. 5, 831-877.

[9] D. Franco, E. Liz, J. J. Nieto, and Y. V. Rogovchenko, A contribution to the study of functional differential equations with impulses, Mathematische Nachrichten 218 (2000), no. 1, 49-60.

[10] M. Frigon and A. Granas, Résultats de type Leray-Schauder pour des contractions sur des espaces de Fréchet, Annales des Sciences Mathématiques du Québec 22 (1998), no. 2, 161-168.

[11] J. R. Graef and A. Ouahab, Some existence and uniqueness results for functional impulsive differential equations in Fréchet spaces, to appear in Dynamics of Continuous, Discrete and Impulsive Systems.

[12] D. Guo, Boundary value problems for impulsive integro-differential equations on unbounded domains in a Banach space, Applied Mathematics and Computation 99 (1999), no. 1, 1-15.

[13] _ Second order integro-differential equations of Volterra type on unbounded domains in Banach spaces, Nonlinear Analysis, Series A: Theory and Methods 41 (2000), no. 3-4, 465-476.

[14] _ A class of second-order impulsive integro-differential equations on unbounded domain in a Banach space, Applied Mathematics and Computation 125 (2002), no. 1, 59-77.

[15] _ Multiple positive solutions for first order nonlinear impulsive integro-differential equations in a Banach space, Applied Mathematics and Computation 143 (2003), no. 2-3, 233-249.

[16] _ Multiple positive solutions for first order nonlinear integro-differential equations in $\mathrm{Ba}$ nach spaces, Nonlinear Analysis 53 (2003), no. 2, 183-195.

[17] D. Guo and X. Z. Liu, Impulsive integro-differential equations on unbounded domain in a Banach space, Nonlinear Studies 3 (1996), no. 1, 49-57.

[18] J. K. Hale and J. Kato, Phase space for retarded equations with infinite delay, Funkcialaj Ekvacioj 21 (1978), no. 1, 11-41.

[19] J. Henderson and A. Ouahab, Local and global existence and uniqueness results for second and higher order impulsive functional differential equations with infinite delay, to appear.

[20] Y. Hino, S. Murakami, and T. Naito, Functional-Differential Equations with Infinite Delay, Lecture Notes in Mathematics, vol. 1473, Springer, Berlin, 1991.

[21] F. Kappel and W. Schappacher, Some considerations to the fundamental theory of infinite delay equations, Journal of Differential Equations 37 (1980), no. 2, 141-183.

[22] V. Lakshmikantham, D. D. Bănov, and P. S. Simeonov, Theory of Impulsive Differential Equations, Series in Modern Applied Mathematics, vol. 6, World Scientific, New Jersey, 1989.

[23] V. Lakshmikantham, L. Z. Wen, and B. G. Zhang, Theory of Differential Equations with Unbounded Delay, Mathematics and Its Applications, vol. 298, Kluwer Academic, Dordrecht, 1994.

[24] Y. Liu, Boundary value problems for second order differential equations on unbounded domains in a Banach space, Applied Mathematics and Computation 135 (2003), no. 2-3, 569-583.

[25] _ Boundary value problems on half-line for functional differential equations with infinite delay in a Banach space, Nonlinear Analysis 52 (2003), no. 7, 1695-1708.

[26] K. G. Mavridis and P. Ch. Tsamatos, Positive solutions for first order nonlinear functional boundary value problems on infinite intervals, Electronic Journal of Qualitative Theory of Differential Equations 2004 (2004), no. 8, 1-18.

[27] A. M. Samoĭlenko and N. A. Perestyuk, Impulsive Differential Equations, World Scientific Series on Nonlinear Science. Series A: Monographs and Treatises, vol. 14, World Scientific, New Jersey, 1995.

[28] K. Schumacher, Existence and continuous dependence for functional-differential equations with unbounded delay, Archive for Rational Mechanics and Analysis 67 (1978), no. 4, 315-335. 


\section{Impulsive functional differential equations}

[29] J. S. Shin, An existence theorem of functional-differential equations with infinite delay in a Banach space, Funkcialaj Ekvacioj 30 (1987), no. 1, 19-29.

[30] B. Yan and X. Liu, Multiple solutions of impulsive boundary value problems on the half-line in Banach spaces, SUT Journal of Mathematics 36 (2000), no. 2, 167-183.

John R. Graef: Department of Mathematics, University of Tennessee at Chattanooga, Chattanooga, TN 37403, USA

E-mail address: john-graef@utc.edu

Abdelghani Ouahab: Department of Mathematics, University of Sidi Bel Abbes, BP 89, 2000 Sidi Bel Abbes, Algeria

E-mail address: ouahab@univ-sba.dz 


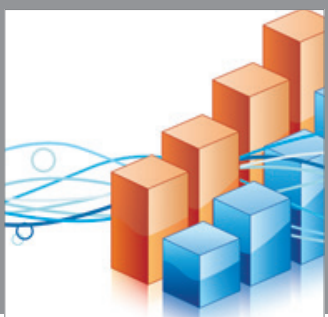

Advances in

Operations Research

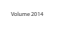

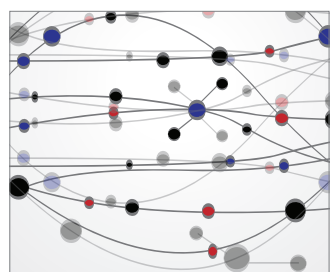

\section{The Scientific} World Journal
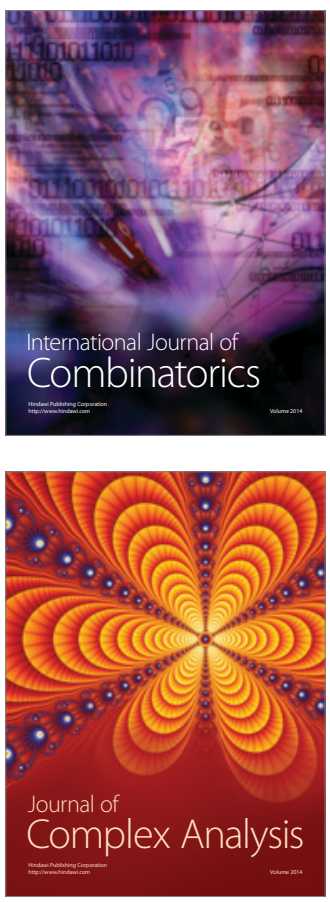

International Journal of

Mathematics and

Mathematical

Sciences
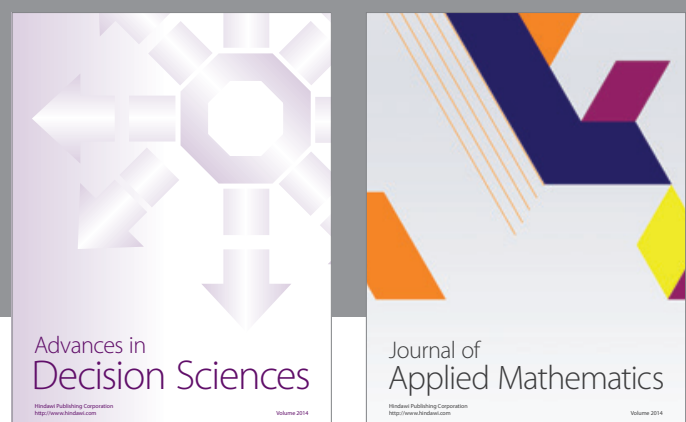

Journal of

Applied Mathematics
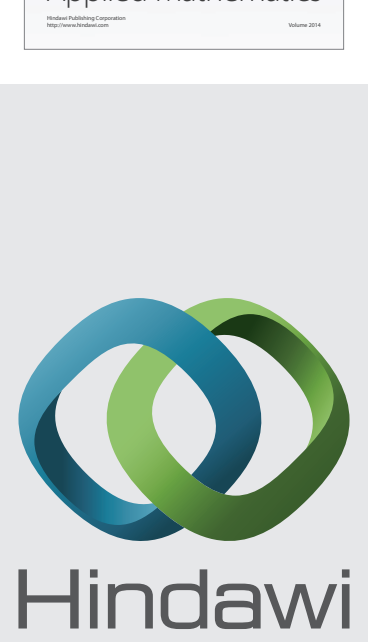

Submit your manuscripts at http://www.hindawi.com
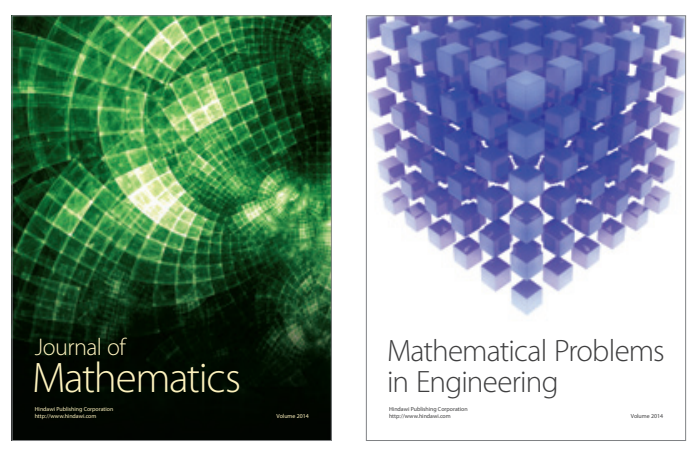

Mathematical Problems in Engineering
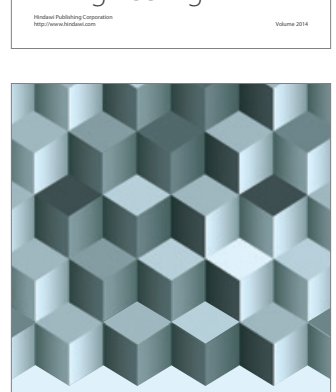

Journal of

Function Spaces
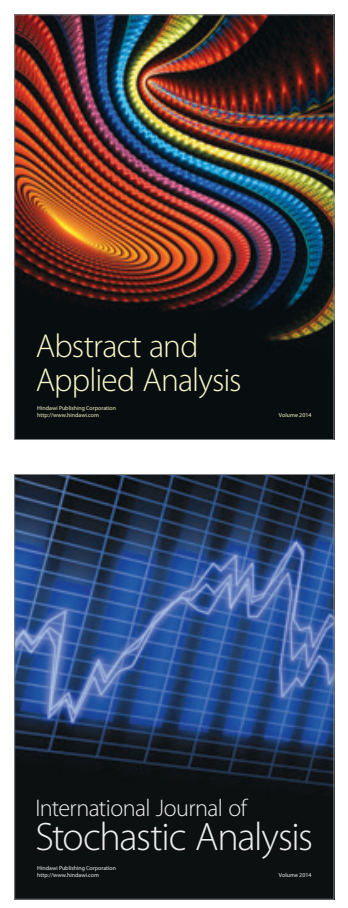

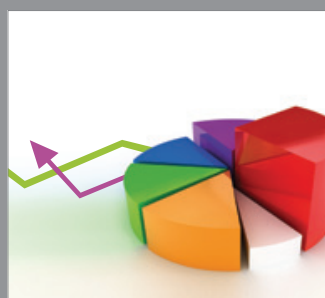

ournal of

Probability and Statistics

Promensencen
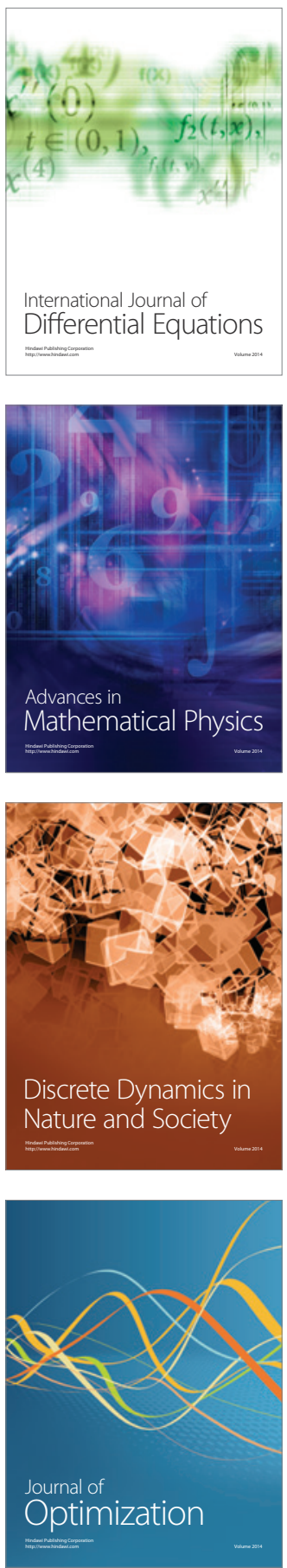